\title{
Removal of nonconstant daily variation by means of wavelet and functional data analysis
}

\author{
I. Maslova, ${ }^{1}$ P. Kokoszka, ${ }^{1}$ J. Sojka, ${ }^{2}$ and L. $\mathrm{Zhu}^{2}$ \\ Received 17 August 2008; revised 14 December 2008; accepted 19 December 2008; published 3 March 2009.
}

[1] We propose a novel approach based on wavelet and functional principal component analysis to produce a cleaner index of the intensity of the symmetric ring current. We use functional canonical correlations to show that the new approach more effectively extracts symmetric global features. The main result of our work is the construction of a new index, which is an improved version of the existing wavelet-based index (WISA) and the old Dst index, in which a constant daily variation is removed. Here, we address the fact that the daily component varies from day to day and construct a "cleaner" index by removing nonconstant daily variations.

Citation: Maslova, I., P. Kokoszka, J. Sojka, and L. Zhu (2009), Removal of nonconstant daily variation by means of wavelet and functional data analysis, J. Geophys. Res., 114, A03202, doi:10.1029/2008JA013685.

\section{Introduction}

[2] It has long been recognized that even on quiet days the daily variation changes very visibly from day to day, both in its amplitude and its shape. This is attributable to multiple dynamic drivers which include not only tidal ionospheric winds, but also the effect of the ChapmanFerraro current, the Sq current, and the magnetotail current [see Xu and Kamide, 2004, and references therein]. On storm days, the effects of these drivers change even more and lead to a very complicated daily variation [Blanc and Richmond, 1980] which is difficult to deconvolute from the global effect of the intensified symmetric ring current. Yet, the $D s t$ and related indices remove a constant daily variation from daily $\mathrm{H}$ component signatures. In the $D s t$, this constant quiet variation is obtained by averaging the daily $\mathrm{H}$ component curves over several quiet days in a month. The main goal of this paper is to introduce a technique allowing to remove daily variations which change from day to day, as well as the effects of other local-time-dependent components, thus leading to an index of storm activity which better reflects the variability of the symmetric ring current.

[3] Our approach builds on the WISA index introduced by Jach et al. [2006] and studied by Xu et al. [2008] and Zhu et al. [2007]. WISA is a 1 min resolution version of the Dst index, and when appropriately averaged, is statistically indistinguishable from the $1 \mathrm{~h}$ Kyoto $D$ st. It enjoys, however, important operational advantages over the Dst. It can be algorithmically computed over time periods as short as two weeks or extending to over a year, and requires only the selection of equatorial terrestrial observatories as input (no selection of quiet days is required). Nevertheless, the WISA

\footnotetext{
${ }^{1}$ Department of Mathematics and Statistics, Utah State University, Logan, Utah, USA.

${ }^{2}$ Department of Physics and Center for Atmospheric and Space Science, Utah State University, Logan, Utah, USA.

Copyright 2009 by the American Geophysical Union. 0148-0227/09/2008JA013685\$09.00
}

procedure also removes a constant daily variation, which is just computed using a different, wavelet-based, algorithm. In order to construct a "cleaner" index of the storm activity, we need to remove the daily variations that are different each day. We propose a general, automatic technique that involves wavelet and principal component analysis methods and extracts a nonconstant daily variation.

[4] This work also builds on the ideas introduced by $X u$ and Kamide [2004], who use the method of natural orthogonal (principal) components to analyze the daily magnetic variation, and argue that the first eigenmode represents the solar quiet daily variation. We address this matter in more detail in section 3. Chen et al. [2007] also use principal component techniques to separate $\mathrm{Sq}$ from complicated disturbances. However, as we show in the following section we believe that the procedure these authors use includes storm features in their estimated daily variation. In our paper we use similar techniques, but we argue that our proposed periodic component estimation methodology is more accurate.

[5] The paper is organized as follows. A brief description of the requisite statistical concepts is provided in section 2 . In section 3, we provide a detailed description of the construction of the improved index. Then, in section 4, we compare the new index to WISA by means of functional canonical correlations. Finally, main conclusions are summarized in section 5 .

\section{Wavelet and Functional Data Analysis}

[6] In this section we first introduce some basic ideas of the wavelet analysis focusing only on the aspects that are relevant to our task. Then we present the functional data analysis, mainly the functional principal component analysis.

[7] First, we introduce a wavelet-based representation of the magnetometer data in order to explain the central ideas of the new procedure. Let, $X_{s}=\left\{X_{s, t}, t=1, \cdots, N\right\}$ be the 
(a) March 29 -- April 3, 2001

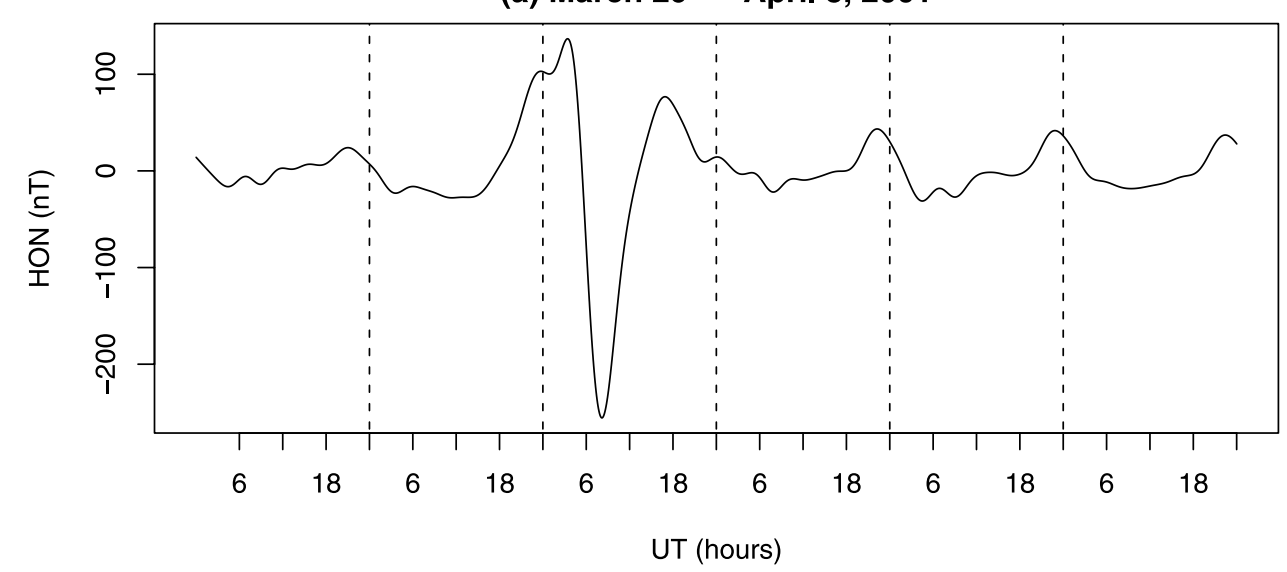

(b) Functional Data, March 29 -- April 3, 2001

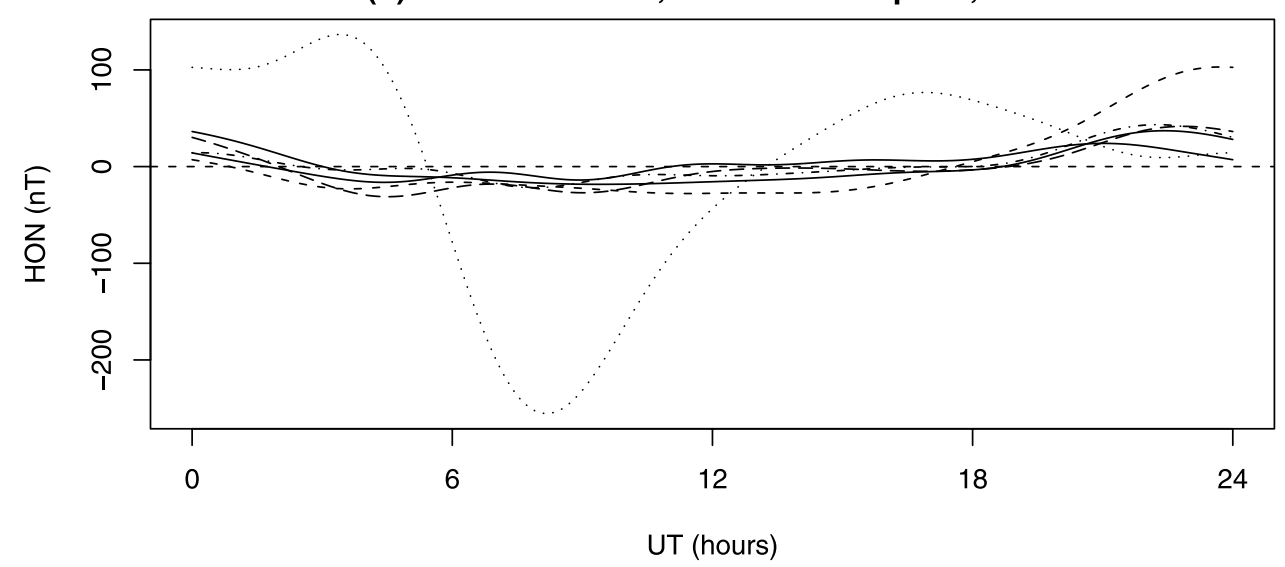

Figure 1. (a) $D_{s, P}$ records, $\mathrm{H}$ component during 29 March to 3 April 2001, HON, UT. (b) Functional data derived from the $\mathrm{H}$ component during 29 March to 3 April 2001, HON, UT.

magnetogram recorded at station $s=1, \cdots, m$, where $N$ is the sample size recorded in minutes. We can write it as

$$
X_{s}(t)=\sum_{j=1}^{J} D_{s, j}(t)+S_{s, j}(t)
$$

where $D_{s, j}=\left\{D_{s, j}(1), \cdots, D_{s, j}(N)\right\}$ are the details, and $S_{s, j}=\left\{S_{s, j}(1), \cdots, S_{s, j}(N)\right\}$ is the smooth. Here, $j=1, \cdots$, $J$ is the multiresolution analysis (MRA) level. The details capture the part of the records that correspond to the frequencies in the range from $2^{-j-1}$ to $2^{-j}$ cycles per minute. For further details, see Percival and Walden [2000, chap. 5]. In this paper, we introduce a procedure that allows us to isolate the storm activity by applying statistical techniques to different levels $j$. We focus on detail levels $j=8,9,10$. As explained by Jach et al. [2006], these are the levels that contain daily periodic features: $j=8$ captures approximately $6 \mathrm{~h}$ periodic component, $j=9$ captures $12 \mathrm{~h}$ component, and $j=10$ captures $24 \mathrm{~h}$ component. We use the same transform and filter as used for WISA construction, i.e., the maximum overlap discrete wavelet transform (MODWT) and the LA(8) filter.

[8] We continue this section by explaining briefly the idea of functional principal component analysis (FPCA);
Ramsay and Silverman [2005, chap. 8] provide a detailed exposition.

[9] Figure 1a shows an example of the data which is the sum of the MRA of magnetometer records at levels $j=8,9$, 10. Dashed lines indicate UT midnight. Our goal is to remove the daily component, so it is natural to split the data into daily observations (functions). The functional observations defined on $24 \mathrm{~h}$ intervals are shown in Figure $1 \mathrm{~b}$. Hence, we treat the daily records as functions and extract the daily variations using FPCA.

[10] In multivariate case we define sets of normalized weights to emphasize types of variation that are most strongly represented in the data.

[11] Let $u_{m}=\left(u_{1 m}, \cdots, u_{p m}\right)^{\prime}$ be the $m$ th weight vector such that $f_{i m}=\sum_{j} u_{j m} x_{i j}$ has the largest mean square $\frac{1}{N} \sum_{i} f_{i m}^{2}$ subject to constraint $\left\|u_{m}\right\|^{2}=1$, and $\sum_{j} u_{j k} u_{j m}=0, k<m$, i.e., each mode must be orthogonal to the previous one so that they are indicating something new. We carry out the procedure, up to a limit of number of variables $p$.

[12] The main idea of the FPCA is to find functions whose inner products with the data yield maximum variation in the curves. FPCA is an orthogonal linear transform that transforms the data to a new coordinate system such that the greatest variance by any projection of the data comes to lie on the first principal component, the second greatest variance comes to lie on the second, and so on. 

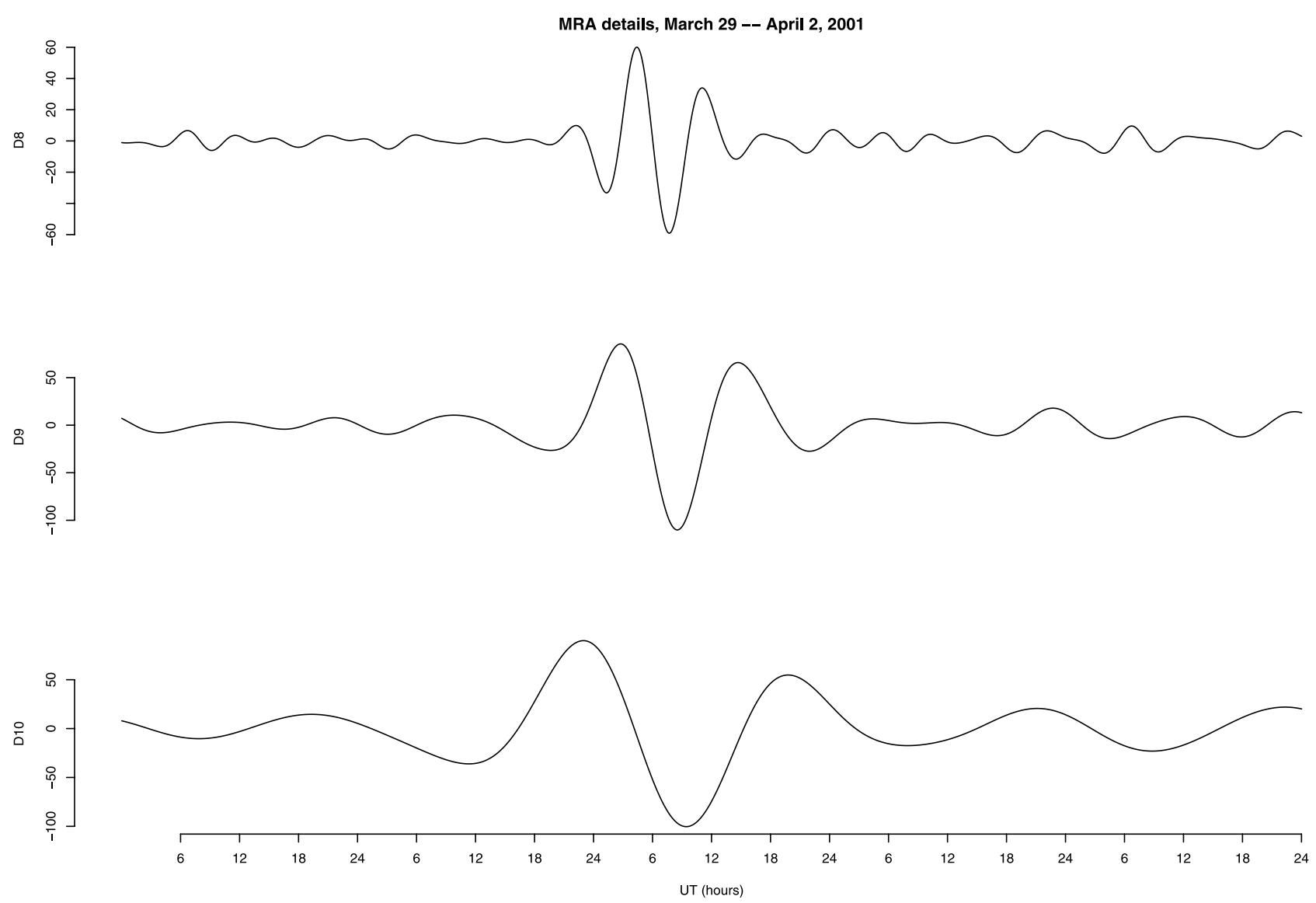

Figure 2. Multiresolution analysis details $D_{8}, D_{9}$, and $D_{10}, 29$ March to 2 April 2001, HON, UT.

[13] In functional context $u(t)$ and $X(t)$ are functions and summation over $j$ is replaced by integration over $t$. Similar to the multivariate case we define the $j$ th principal component score of $X_{i}$ as $\gamma_{j}=\int X_{i}(t) u_{j}(t) d t$. It can be interpreted as the weight of the contribution of the FPC $u_{j}$ to the curve $X_{i}$. Each principal component, say $j$ th $u_{j}(t)$ is chosen to maximize $\frac{1}{N} \sum_{i}\left(\int u_{j}(t) X_{i}(t)\right)^{2}$ subject to constraint: $\int u_{j}^{2}(t) d t=1$. Same as for multivariate PCA, the weight function $u_{j}(t)$ is required to satisfy the orthogonality constraint $\int u_{k}(t) u_{j}(t)$ $d t=0, k<j$.

[14] Principal components form an orthonormal system. The main goal of the FPCA is to find the dominant modes of variation in the data. In this study we are interested in the first principal component. Xu and Kamide [2004] and Chen et al. [2007] argue that it captures the main features of the daily $\mathrm{Sq}$ variation. However, our approach differs from the one introduced by $X u$ and Kamide [2004]. Instead of the raw data, we use filtered records, i.e., the sum of three levels of MRA, and remove storm features with care.

\section{Removal of the Daily Variation}

[15] In this section we provide the details on the removal of the daily component from the magnetometer data. During quiet periods, it is basically the Sq variation, but during disturbed periods it may reflect the dynamo effect and disturbances from other M-I currents. This component is semiperiodic, as it is caused by the rotation of the Earth. The atmospheric dynamo generates currents that flow in the upper atmosphere in the E region. These currents arise as a consequence of atmosphere storm dynamics (winds) which have been generated by geomagnetic storms. During storms these wind patterns are quite different from the quiet time winds that create the Sq current system.

[16] The periodicity is clearly visible in MRA details $D_{s, j}$ for levels $j=8,9,10$ (see Figure 2). However, one can also see that it is enhanced during a storm. This fact is taken into account while removing the daily variation from storm features.

[17] Let

$$
D_{s, P}(t)=D_{s, 8}(t)+D_{s, 9}(t)+D_{s, 10}(t), t=1, \cdots, N
$$

be the part of the signal that includes practically all frequencies of the daily component spectrum. The subscript "P" stands for (semi) "periodic."

[18] Our goal is to extract the signature of the storm activity from the $D_{s, P}$. A storm is a global event and it is visible in the records of all stations. Its signatures at various stations are aligned in UT. Figure 3 illustrates the fact that the storm features are roughly aligned in UT, whereas the periodic components are not. Therefore, we want to extract as many UT aligned features as possible. Such features are attributable to the storm activity, and should be included in the index.

[19] As mentioned above, the periodic daily component is a local feature that is approximately aligned in LT. In order 
MRA, Dsp, March 29 - April 2, 2001

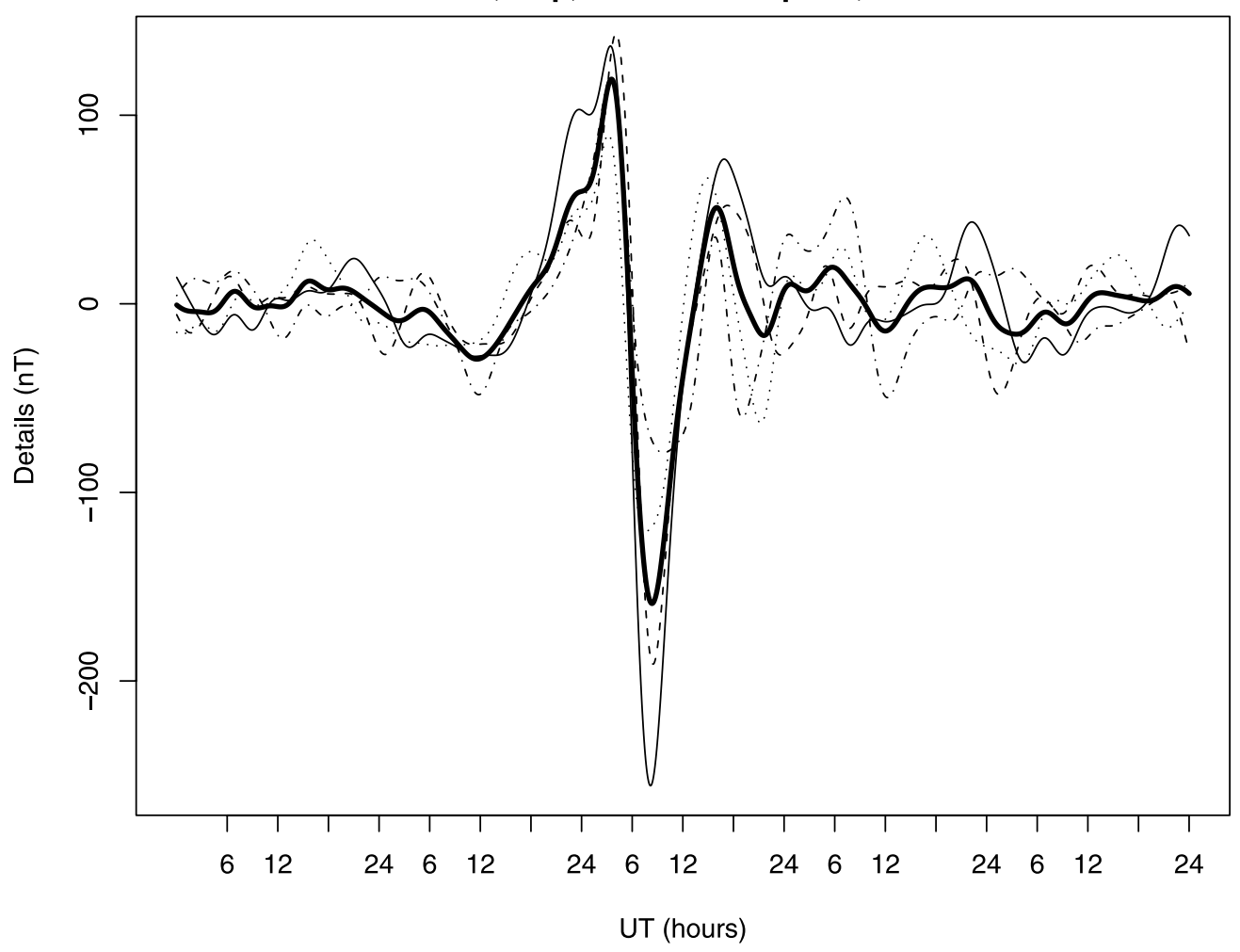

Figure 3. $D_{s, P}$ components and their mean (thick line) of 4 Dst stations, HON, KAK, SJG, and HER, during disturbed period of time: 29 March to 2 April, UT.

to separate it from the storm signature, we first need to remove all features aligned in UT. Therefore, we remove

$$
\bar{D}_{P}(t)=\frac{1}{m} \sum_{s=1}^{m} D_{s, P}(t), t=1, \cdots, N
$$

which is the average of $D_{s, P}$ of all stations $s=1,2, \cdots, m$ used in the study. The mean $\bar{D}_{P}$ roughly follows the storm pattern (see the thick line in Figure 3). After the mean removal, the data is mostly cleaned from events aligned in UT (see Figure 4) and so the daily variation can be removed more effectively. We emphasize, that the computation of $\bar{D}_{P}(t)$ is merely a preliminary step. As the records from different stations may have slightly different dynamic ranges, the average may be biased toward some stations. Averaging with appropriately computed weights is possible, but this increases the complexity of the algorithm, and leads to negligible gains.

[20] Denote by $D_{s, P}^{c}(t)=D_{s, P}(t)-\bar{D}_{P}(t)$ the centered record at station $s$. Figures 5 (disturbed period of time) and 6 (quiet time) show that $D_{S, P}^{c}$ contains a strong quasiperiodic component which reflects the $\mathrm{Sq}$ variation during quiet periods and a more complicated $\mathrm{Sq}$ variations during storm periods. In Figure 5 we see that there is nighttime activity in $D_{s, P}^{c}$. We want to add it to the storm index, but not the quasiperiodic component. We therefore postulate that

$$
D_{s, P}^{c}(t)=P_{s}(t)+R_{s}(t), t=1, \cdots, N,
$$

where $P_{s}$ is identified with the daily periodic component and $R_{S}$ is the remaining effect of a storm left after the average removal. Next, we apply principal component analysis techniques to estimate the daily variation $P_{s}$. We convert $D_{s, P}^{c}$ into functional object, i.e., daily functions that start at UT midnight. Using principal component analysis we can write $\left(t^{\prime}\right.$ is the time in minutes within 1 day)

$$
D_{s, P}^{c}\left(t^{\prime}\right)=\mu_{s}\left(t^{\prime}\right)+\sum_{j=1}^{\infty} \gamma_{s, j} u_{s, j}\left(t^{\prime}\right), t^{\prime}=1, \cdots, 1440,
$$

where $\mu_{s}\left(t^{\prime}\right)$ is the daily mean, $\gamma_{s, j}$ is a score vector for $j$ th $\mathrm{PC}$, and $u_{s, j}$ is the $j$ th $\mathrm{PC}$ for station $s$. We assume that periodic component for day $i=1, \cdots, N / 1440$ is

$$
P_{s, i}\left(t^{\prime}\right)=\mu_{s}\left(t^{\prime}\right)+\gamma_{s, 1, i}^{*} u_{s, 1}\left(t^{\prime}\right), t^{\prime}=1, \cdots, 1440,
$$

where $\gamma_{s, 1, i}^{*}$ is a filtered score for the $i$ th day described below. The function $u_{s, 1}\left(t^{\prime}\right)$ is the first PC for station $s$. In (2) $\mu_{s}\left(t^{\prime}\right)$ and $u_{s, 1}\left(t^{\prime}\right)$ are deterministic functions defined over the 24-h interval, and $\gamma_{s, 1}^{*}$ are random weights that change from day to day. Hence, the extracted daily component $P_{s}(t)$ is nonconstant. Note that $P_{s, i}\left(t^{\prime}\right)$ where $t^{\prime}=1, \cdots, 1440$ and $i=1, \cdots, N / 1440$ is the same daily periodic component as $P_{s}(t)$ where $t=1, \cdots, N$ split into daily functions.

[21] Decomposition (2) is akin to the ideas of $X u$ and Kamide [2004] and Chen et al. [2007], who argued that the first principal component follows the pattern of the daily $\mathrm{Sq}$ variation. However, while these authors work with the raw 


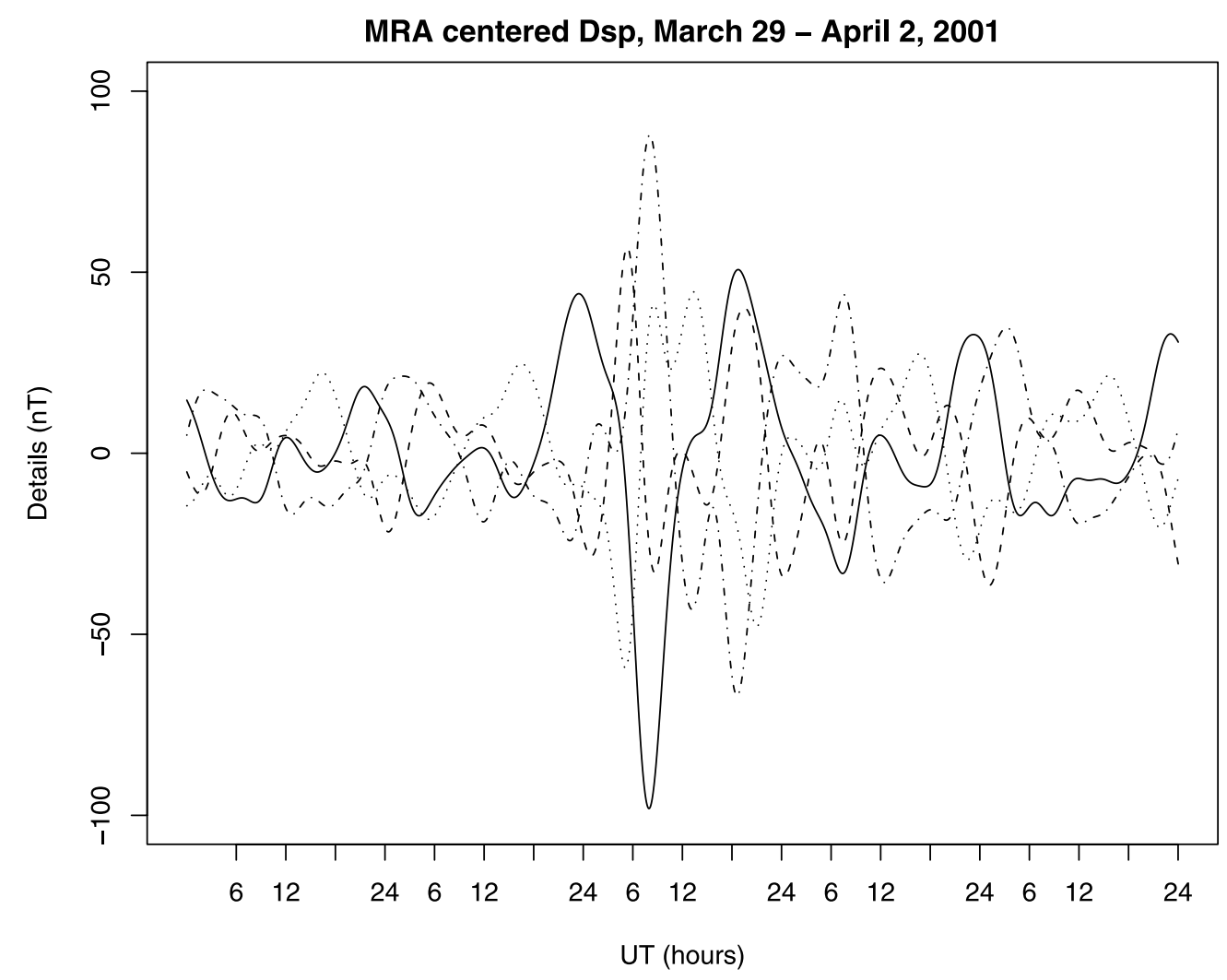

Figure 4. Centered $D_{s, P}$ components of 4 Dst stations, HON, KAK, SJG, and HER, during quiet time: 29 March to 2 April, UT.

magnetometer records, we first apply a wavelet filter to the data and use just the levels that contain the periodic component. We also remove the average of several stations to separate the storm effect. So, in our paper, to estimate daily periodic component $P_{s}$, we compute the first PC of $D_{S, P}^{c}$ rather than the first PC of the raw magnetometer data.

[22] As mentioned earlier, the three selected MRA levels contain residual storm features. Averaging over $m$ stations removed a substantial part of them, but not all. Daily scores of the first PC, $\gamma_{s, 1}$, show extreme values during the days when a storm occurred. Therefore, they contain the residual signature of the storm which should be added to the index.

[23] Let $p_{0.95, s}$ denote the 95th percentile of the daily scores $\gamma_{s, 1}$ for station $s$. We define

$$
\gamma_{s, 1}^{*}=\left\{\begin{array}{cl}
M_{\gamma_{s, 1},}, & \text { if }\left|\gamma_{s, 1}\right|>p_{0.95, s} \text { for all } s \\
\gamma_{s, 1}, & \text { otherwise }
\end{array}\right.
$$

where $M \gamma_{s, 1}$ is the median score of station $s$. This means that to extract the residual storm effect from the daily scores we find the largest $5 \%$ of the scores $\gamma_{s, 1}$ for each station $s$ individually. If the extreme value is captured by all stations we replace it by the median score, $M \gamma_{s, 1}$, of the corresponding station. The scores defined in (3) are used to compute daily periodic component $P_{s}$ defined in (2).

[24] Therefore, the residual storm contribution is

$$
R_{s}(t)=D_{s, P}^{c}(t)-P_{s}(t), t=1, \cdots, N
$$

[25] It allows us to construct a so-called preindex, which consists of a storm signature extracted from three MRA levels of station $s$ magnetogram. It is defined as follows

$$
I_{s}(t)=\bar{D}_{P}(t)+R_{s}(t), t=1, \cdots, N
$$

and is the contribution of station $s$ to the storm signature. An index is constructed by averaging the $I_{S}(t)$ from judiciously selected stations.

[26] We now provide a brief summary of the procedure introduced in this section.

[27] 1. Perform the MRA on the raw magnetometer records, and extract details at levels $j=8,9,10$ using MODWT and LA(8) filter. From here, work with the sum of these three levels of details.

[28] 2. Find the average of all stations used in the study, and remove it from the record at each station.

[29] 3. Convert the data from previous step into a functional 1-day object. Compute the first FPC.

[30] 4. Replace the outlier scores aligned through all stations with median scores. Use those scores to estimate the daily component.

[31] 5. Compute the storm activity preindex.

\section{Comparison of Indices}

[32] The objective of this section is to compare the improved preindex introduced in section 3 to the known WISA preindex, i.e., the part of the storm activity extracted from the three MRA levels. 

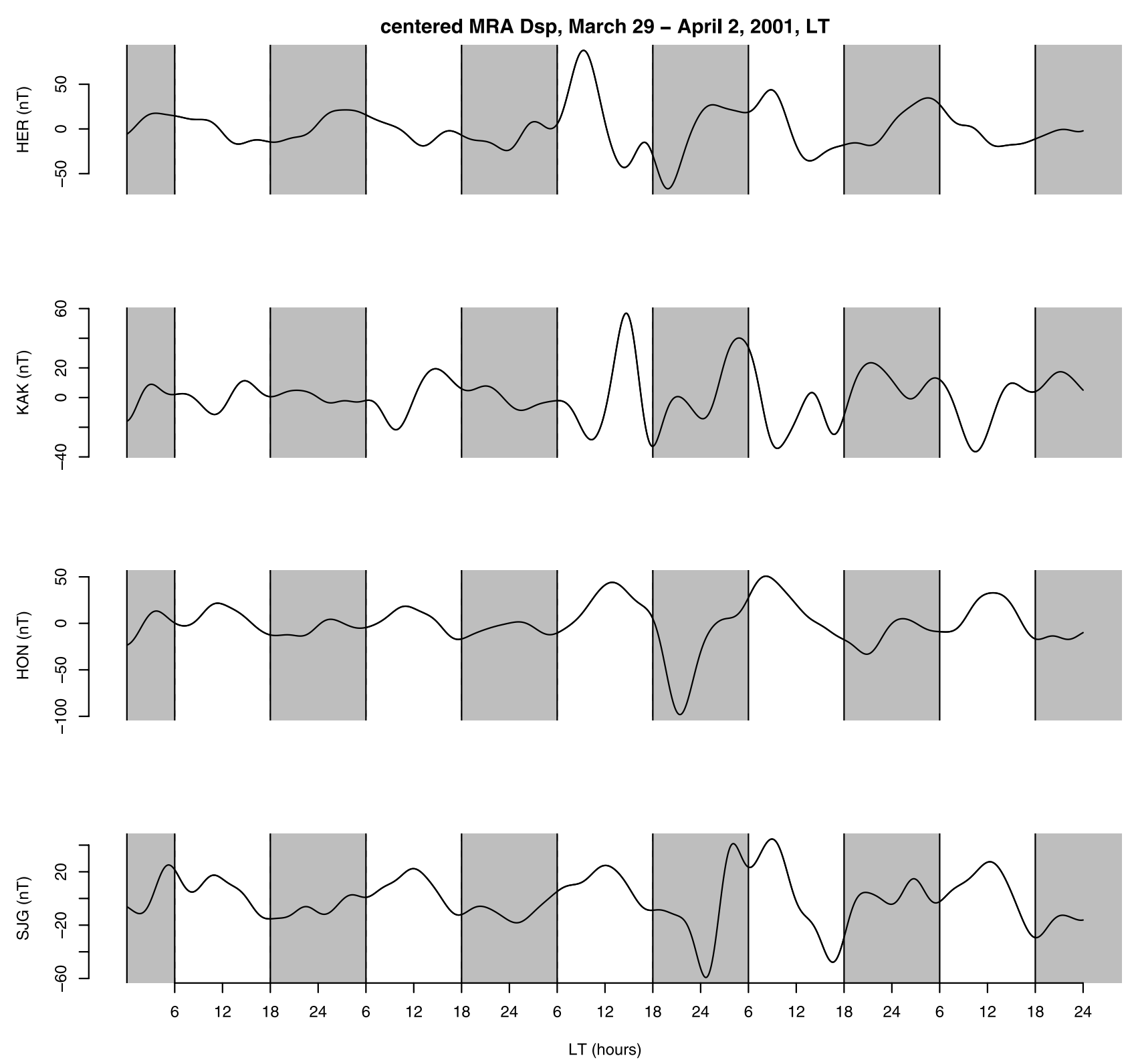

Figure 5. $D_{s, P}^{c}$ components of 4 Dst stations: HON, KAK, SJG, and HER, during disturbed period of time: 29 March to 2 April, in LT. Grey areas correspond to nighttime.

[33] First, we describe the data sets we use in this study. Then, we introduce a quantitative procedure we apply to compare the new preindex to the WISA preindex.

[34] We use the $\mathrm{H}$ component of the magnetometer records, the same as in WISA and Dst. Table 1 contains the list of the stations used in this study. To verify our results we use two four-station combinations and one sixstation combination (see Table 2). The stations in each combination are roughly equispaced in the equatorial zone. The first set of four stations, HER, KAK, HON, SJG, was used because it is the standard Dst set, even though the stations HER, PHU, HON, SJG, are more equispaced. The index and the canonical correlations for the two sets do not differ significantly. It is in fact an advantage of our method that it is fully automatic, and records from any set of stations can be used as inputs, and the resulting indices compared.

[35] In order to produce a comprehensive study we applied the new procedure to 3,5 , and 6 month periods during 2001.

[36] Figure 7 provides a visual comparison of the improved preindex to the data, $D_{s, P}$, it was constructed from. During the disturbed periods of time (Figure 7, top) improved preindex captures the storm signature (solid line), and most of the daily periodic component is eliminated from it during the quiet periods (Figure 7, bottom). What is left is in the range of $\pm 10 \mathrm{nT}$, slightly above the measurement error. During quiet days the original data are semiperiodic, so any index is semiperiodic, but with a smaller amplitude and often out of phase with the data. 

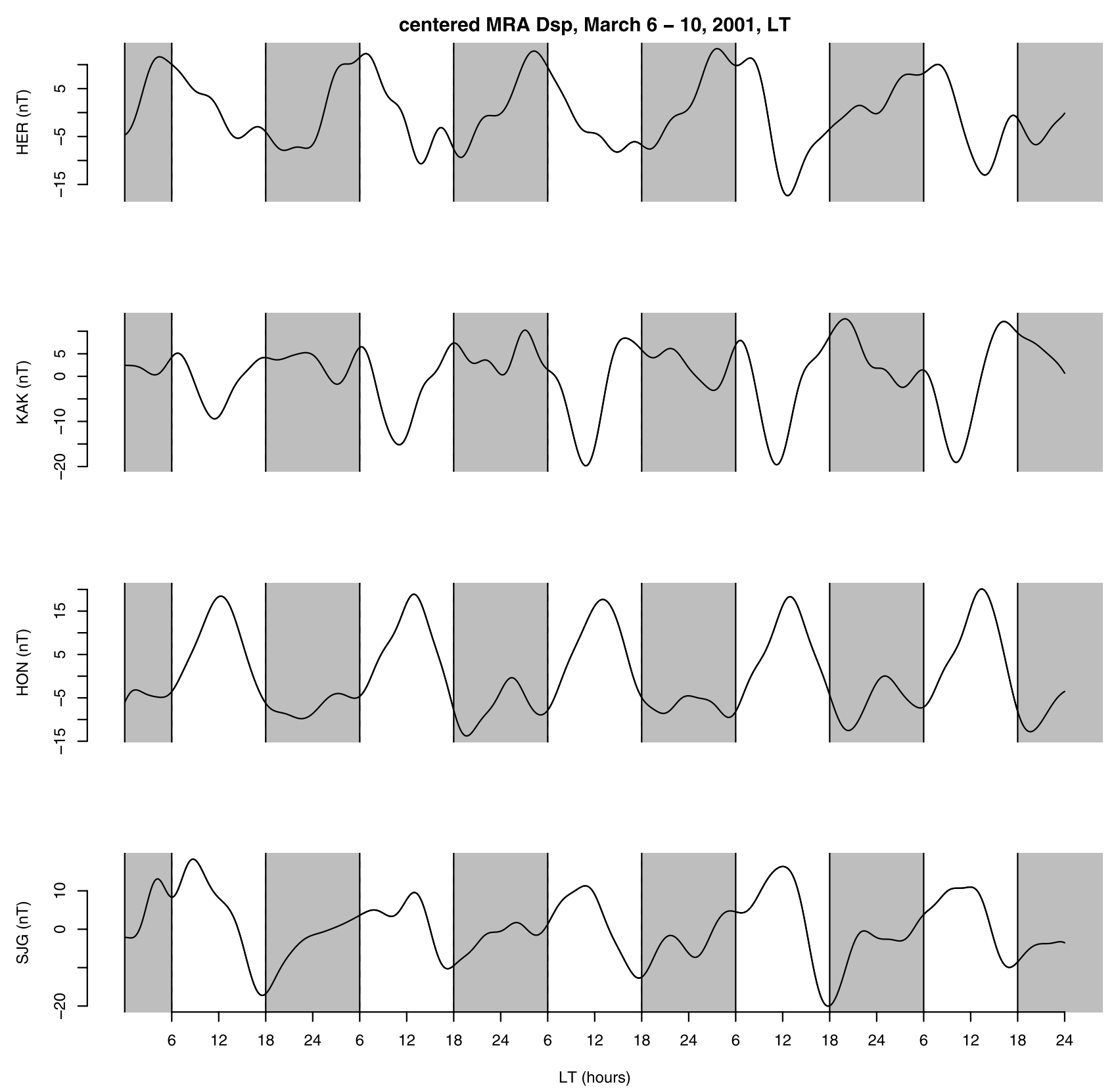

Figure 6. $D_{s, P}^{c}$ components of 4 Dst stations: HON, KAK, SJG, and HER, during quiet time: 6-10 March, in LT. Grey areas correspond to nighttime.

[37] A visual comparison is not enough to conclude that our proposed method removes daily periodic activity better than WISA or Dst. Therefore, we propose to use the functional canonical correlations to evaluate level of improvement quantitatively.

Table 1. Geomagnetic Observatories Used in This Study

\begin{tabular}{clcr}
\hline Station & \multicolumn{1}{c}{ Name } & Colatitude & Longitude \\
\hline 1 & Hermanus (HER) & 124.43 & 19.23 \\
2 & Antananarivo (TAN) & 108.92 & 47.55 \\
3 & Phuthuy (PHU) & 68.97 & 105.95 \\
4 & Kakioka (KAK) & 53.77 & 140.18 \\
5 & Honolulu (HON) & 68.68 & 202.00 \\
6 & San Juan (SJG) & 71.89 & 293.85 \\
7 & Mbour (MBO) & 75.62 & 343.03 \\
\hline
\end{tabular}

\subsection{Functional Canonical Correlations}

[38] In this section we introduce the main idea of the functional canonical correlation analysis (FCCA).

[39] Classical canonical correlation analysis (CCA) computes linear transformations of two variables such that the correlation between the transformed variables is maximized. Let $\left(x_{i}, y_{i}\right), i=1, \cdots, n$, be pairs of observed vectors. The

Table 2. Combinations of Four and Six Stations Used to Test the New Method

\begin{tabular}{ll}
\hline Set & \multicolumn{1}{c}{ Station } \\
\hline Four-1 & HER, KAK, HON, SJG \\
Four-2 & TAN, KAK, HON, SJG \\
Six-1 & TAN, PHU, KAK, HON, SJG, MBO \\
\hline
\end{tabular}




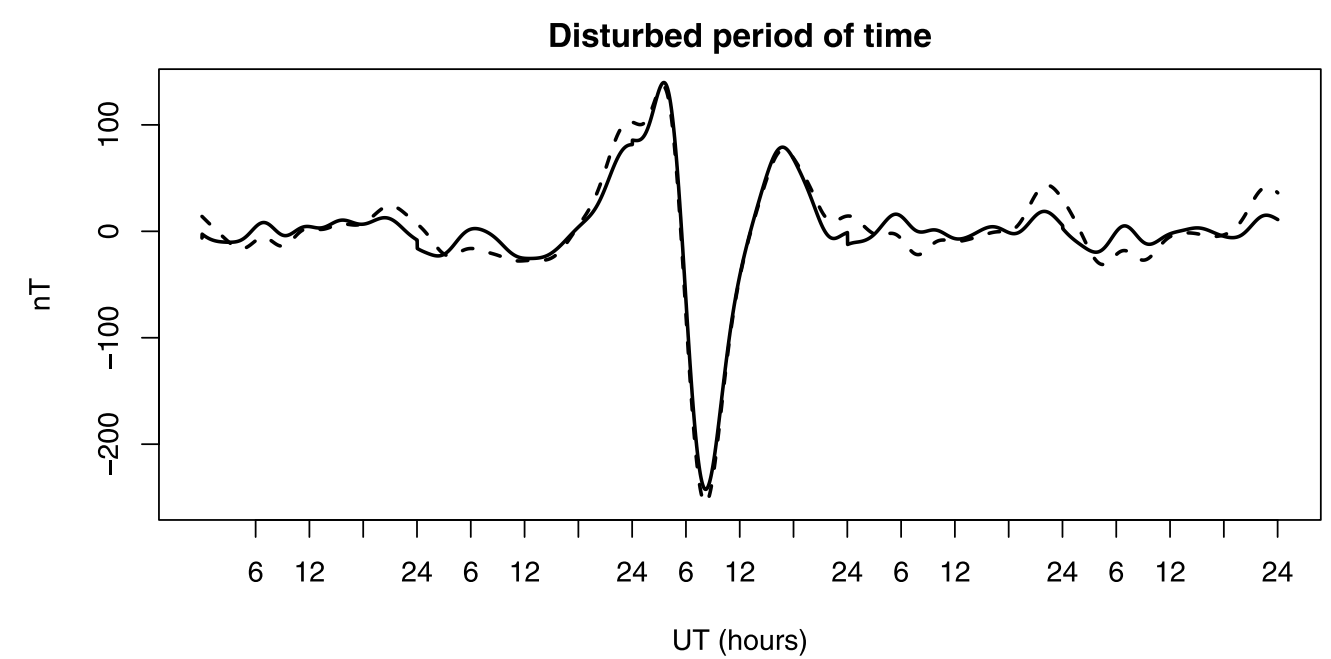

Quiet period of time

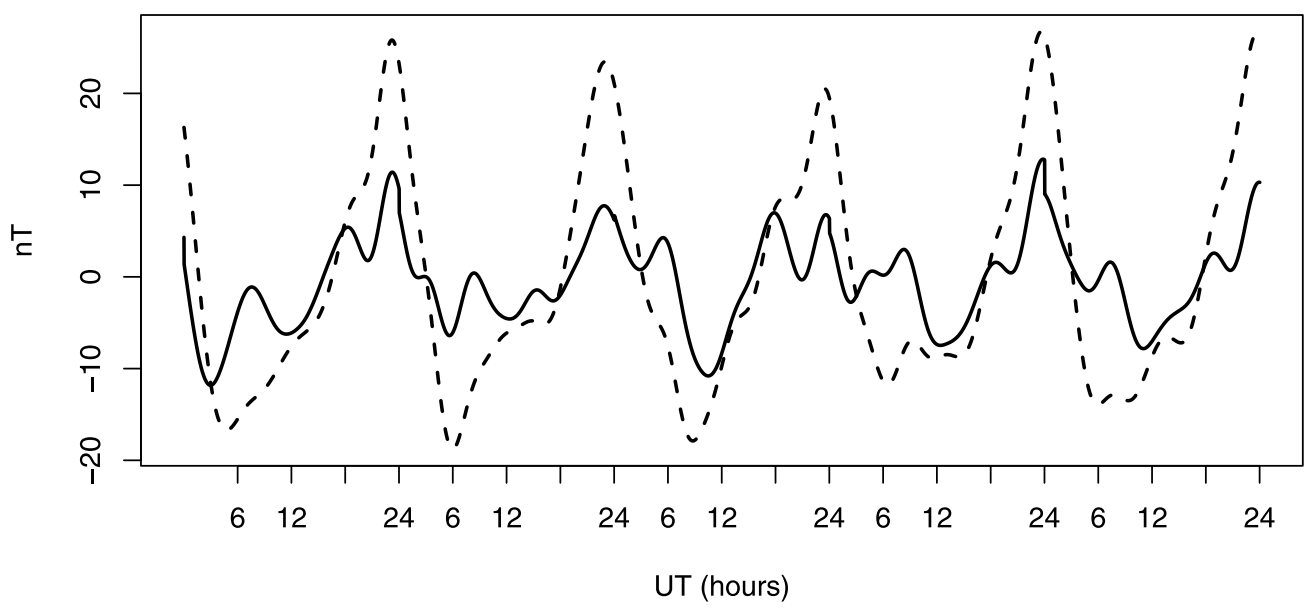

Figure 7. Improved preindex (solid line) and $D_{s, P}$ (dashed line) for HON station during (top) disturbed and (bottom) quiet periods.

goal is to find vectors $a_{1}$ and $b_{1}$ such that the correlation between linear combinations $a_{1}^{\prime} x_{i}$ and $b_{1}^{\prime} y_{i}$ is the highest. A detailed discussion on CCA is given by Anderson [1984, chap. 12].

[40] Functional CCA provides a similar tool for investigating the relationship of the variability of functions. It helps to identify the modes of variability in the two sets of curves that are associated with each other most strongly.

[41] Suppose, we observe $N$ pairs of curves $\left(X_{i}(t), Y_{i}(t)\right)$. Let $(\xi, \eta)$ denote canonical variate weight functions defined such that the correlation between canonical variates $\int \xi X_{i}$ and $\int \eta Y_{i}$ is the highest. As first observed by Leurgans et al. [1993] and discussed by Ramsay and Silverman [2005, chap. 11], to find a meaningful correlation an appropriate smoothing is essential. We are interested in comparing the canonical correlations for different methods and different data sets, therefore, we used the same smoothing parameter for all the data. The choice of the smoothing parameter does not change the overall conclusion.

[42] In our application, the $X_{i}$ represent the daily preindex curves at one station (e.g., HON) and $Y_{i}$ the preindex curves at another station (e.g., HER). These curves can be com- puted using our method or WISA. If the canonical correlation for the curves computed using the new method is higher than for the curves obtained from the WISA procedure, it means that the new method isolates more features that are common for the $X_{i}$ and the $Y_{i}$, e.g., for HON and HER.

[43] The next section describes such comparison.

\subsection{Quantitative Comparison of Different Methodologies}

[44] In this section we present the results of the comparison of preindexes produced using the new improved method and WISA. We do not perform any direct comparison to the Dst. However, since WISA is statistically indistinguishable from Dst [see Jach et al., 2006], the conclusions we make about the WISA apply to the Dst index as well.

[45] A method produces a "cleaner" preindex (and index eventually) if local features are removed in a more efficient way. Therefore, the association of the preindexes of different stations should be strong. Using FCCA we show that our new technique isolates the effect of a storm better than the WISA procedure. 

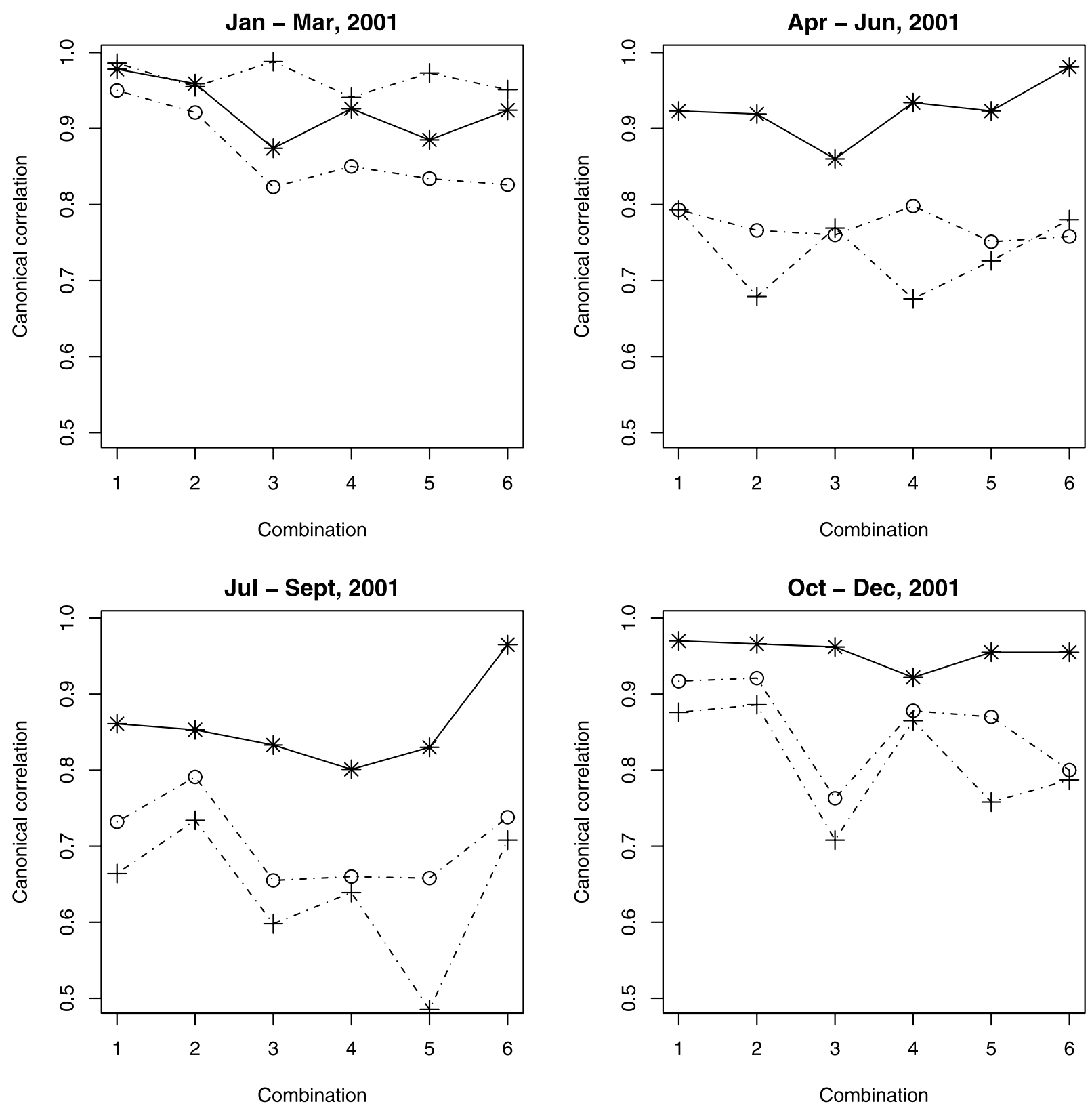

Figure 8. Canonical correlations for the new method (star), new method without centering (cross), and WISA (circle), applied to all combinations of four Dst stations (see Table 3). 

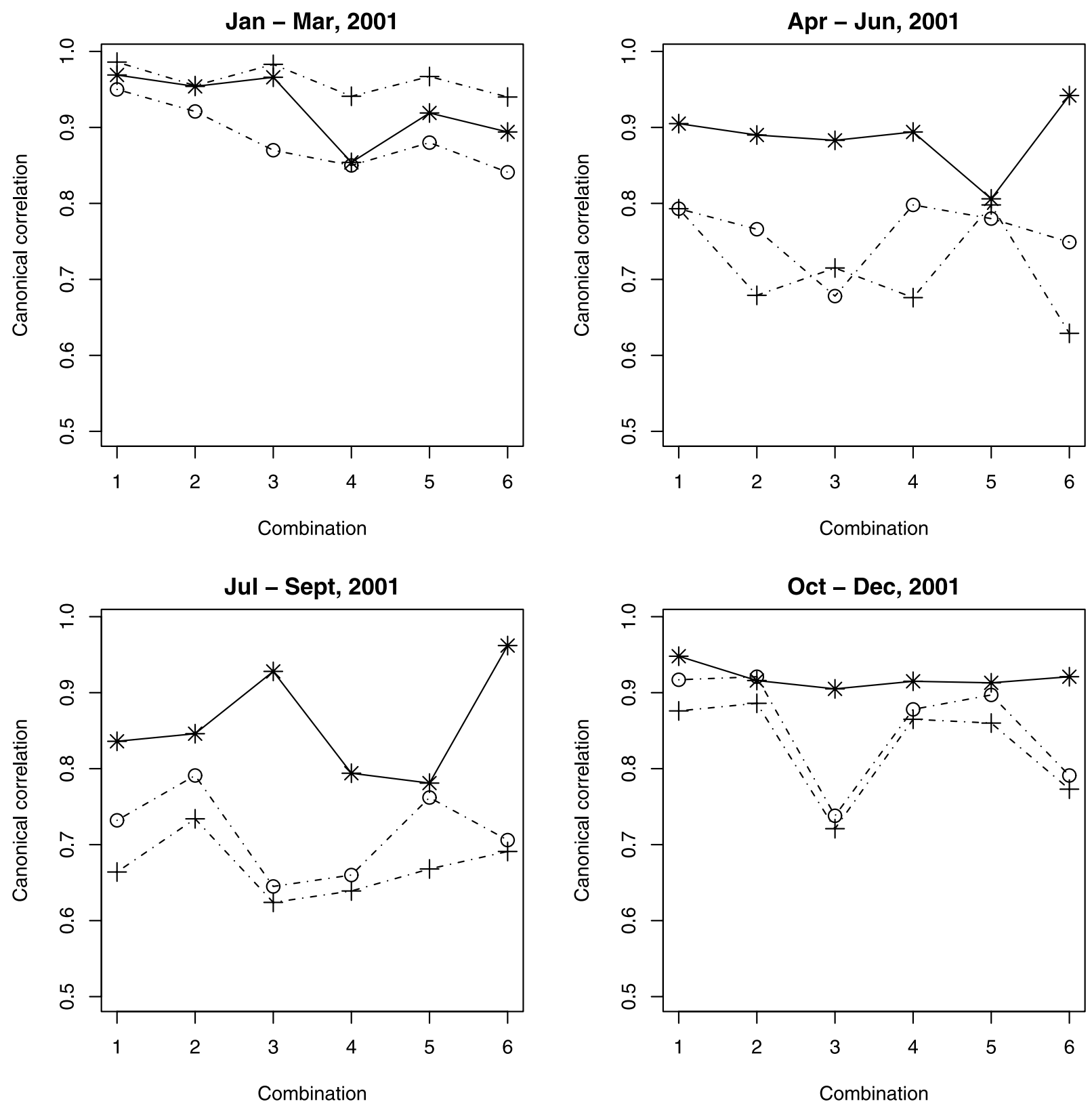

Figure 9. Canonical correlations for the new method (star), new method without centering (cross), and WISA (circle), applied to all combinations of second set of four stations (see Table 4). 

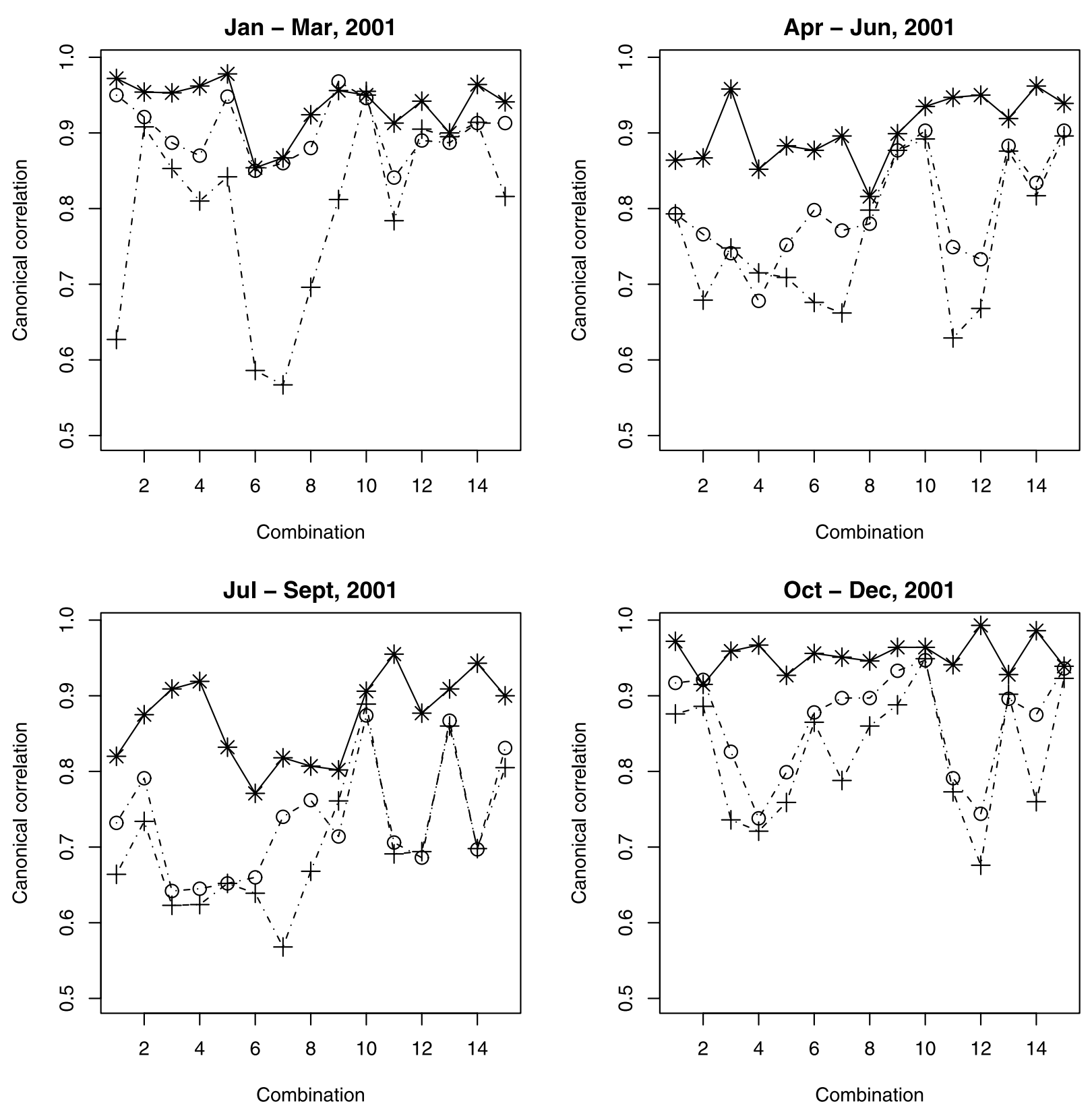

Figure 10. Canonical correlations for the new method (star), new method without centering (cross), and WISA (circle), applied to all combinations of six stations (see Table 5). 

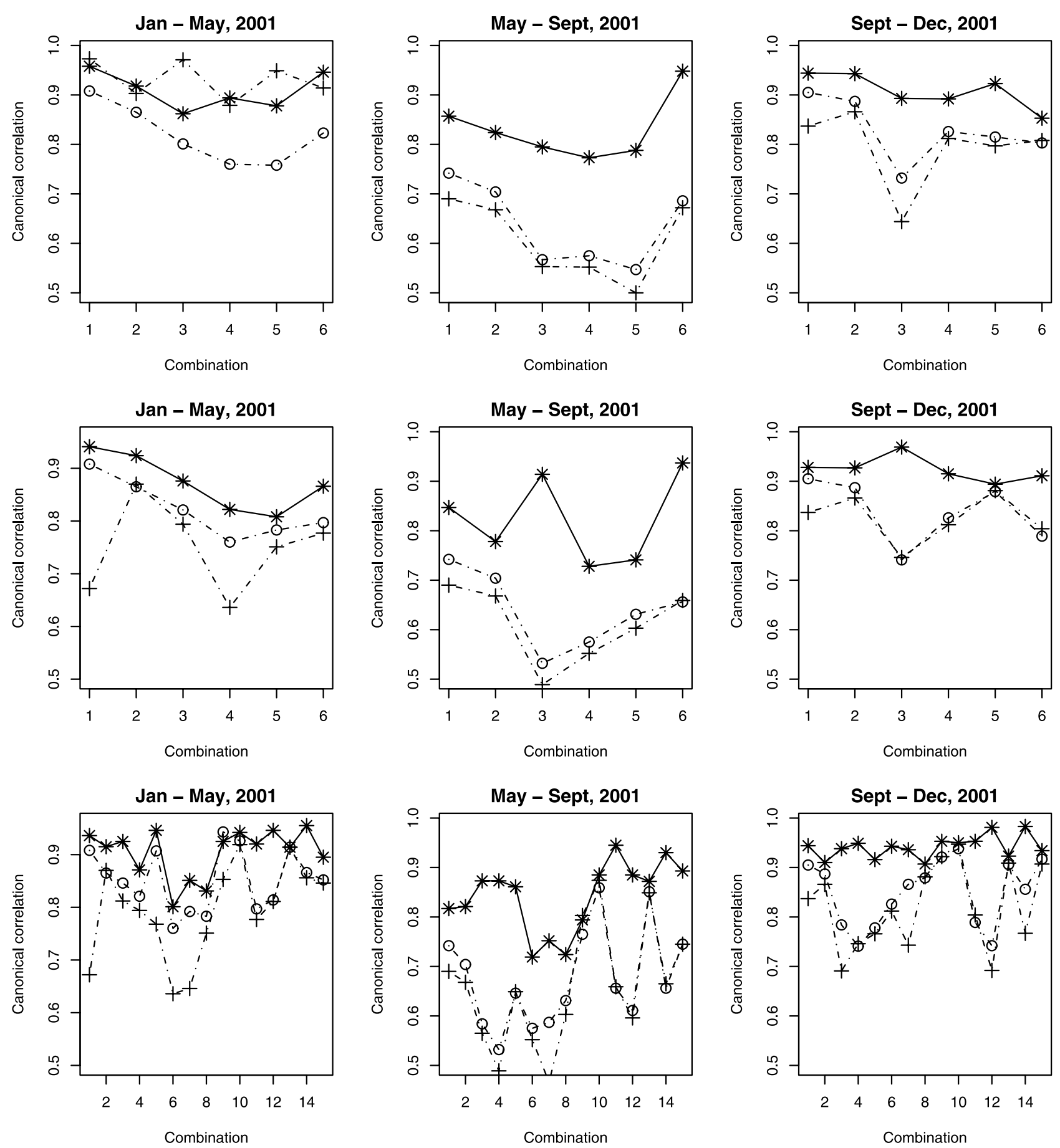

Figure 11. Canonical correlations for the new method (star), new method without centering (cross), and WISA (circle), applied to all combinations of (top) the first set of stations (combinations are given in Table 3), (middle) the second set of stations (combinations are given in Table 4), and (bottom) the third set of stations (combinations are given in Table 5). 
(a) Jan - Jun, 2001

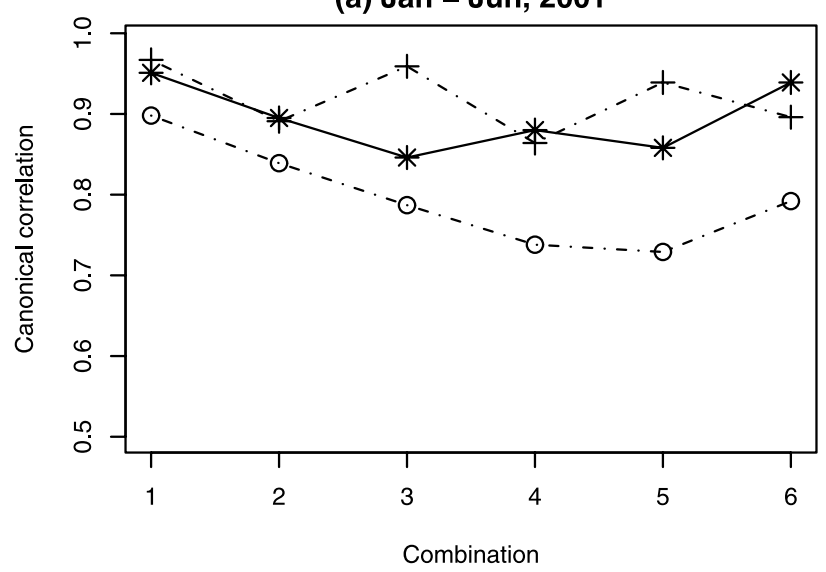

(c) Jan - Jun, 2001

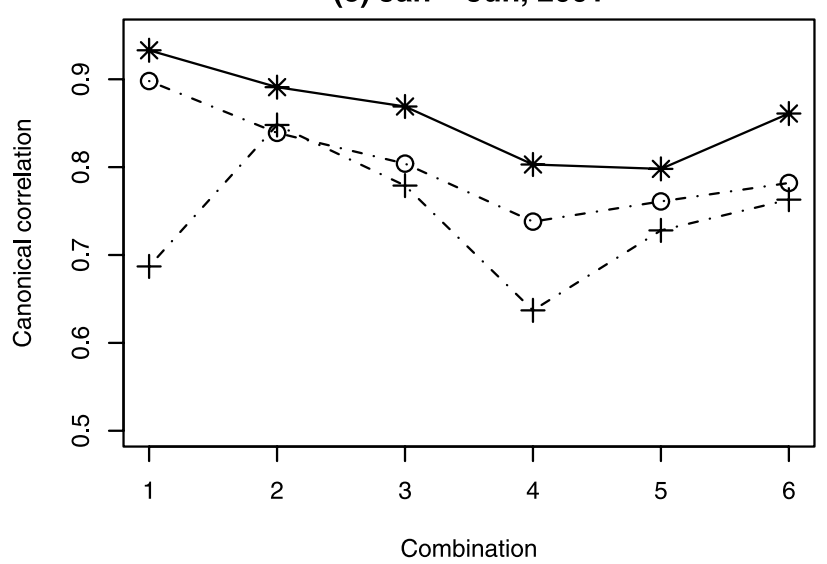

(e) Jan - Jun, 2001

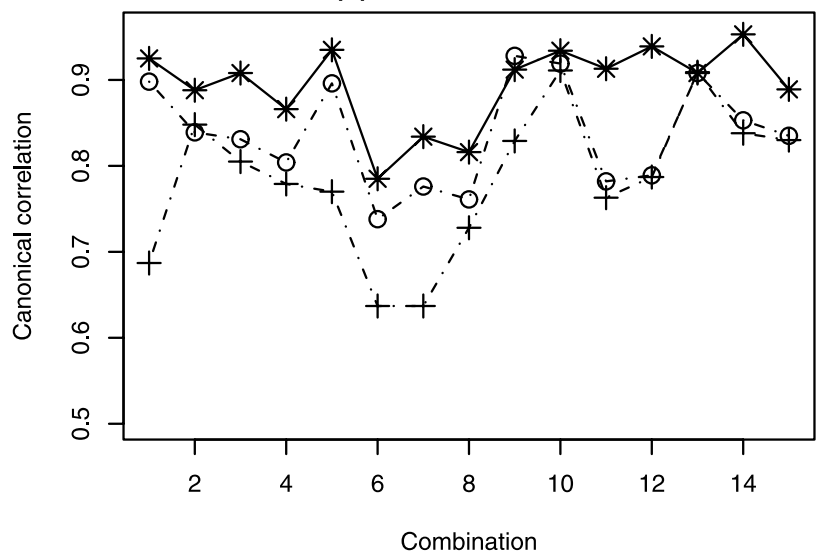

(b) Jun - Dec, 2001

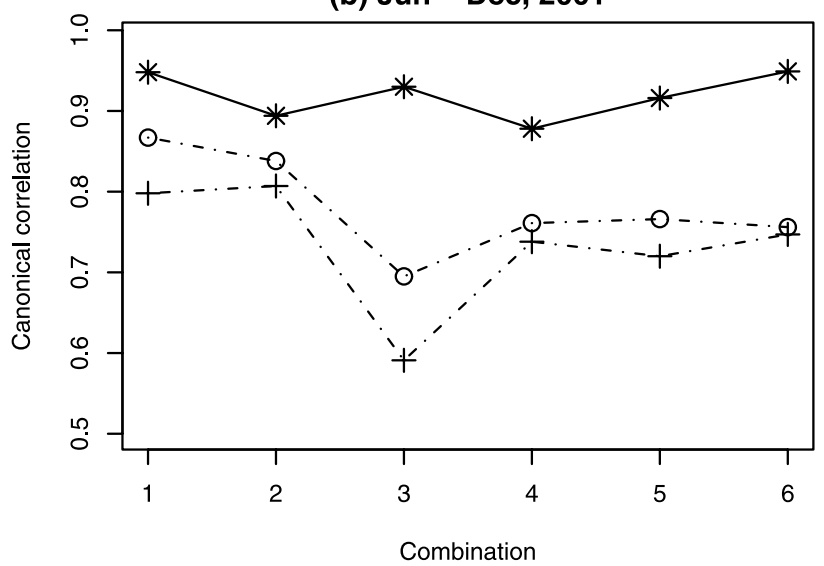

(d) Jun - Dec, 2001

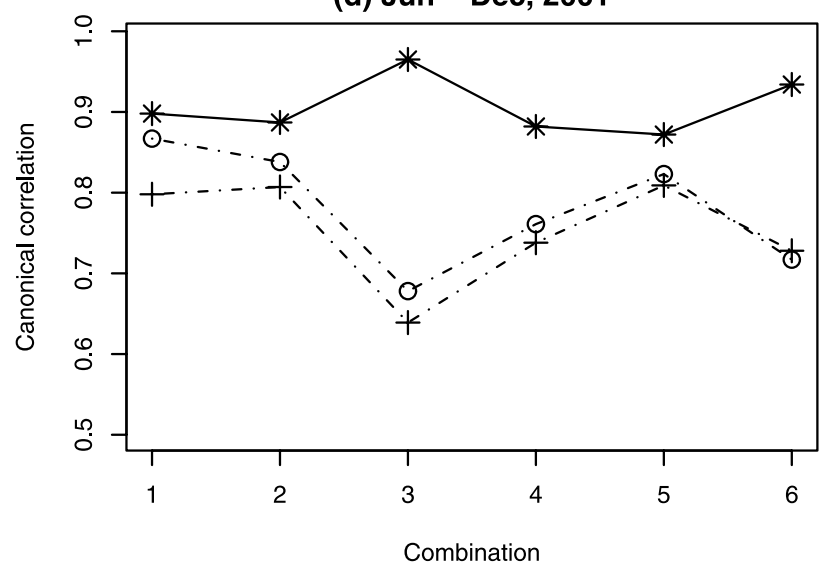

(f) Jun - Dec, 2001

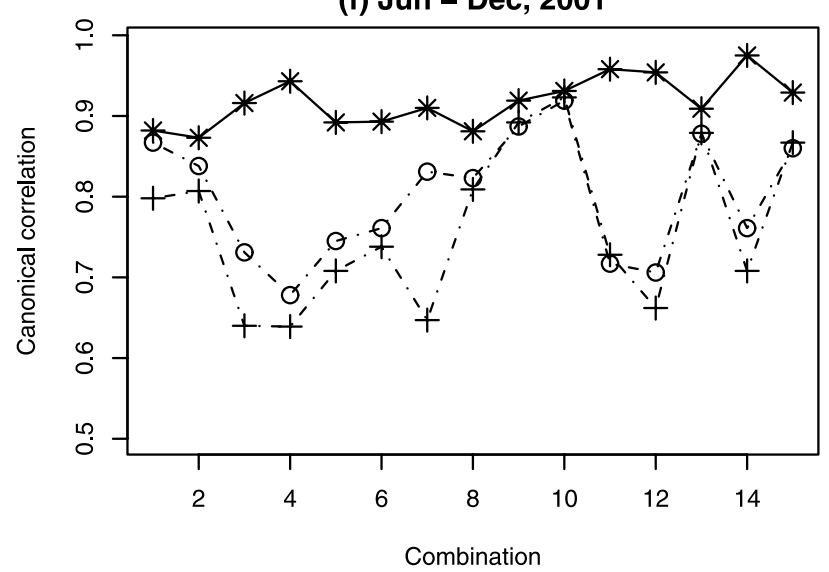

Figure 12. Canonical correlations for the new method (star), new method without centering (cross), and WISA (circle), applied to all combinations of (top) the first set of stations (combinations are given in Table 3), (middle) the second set of stations (combinations are given in Table 4), and (bottom) the third set of stations (combinations are given in Table 5). 
Table 3. Combinations of Four Dst Stations (First Set) Used to Compare Methodologies

\begin{tabular}{cl}
\hline Combination & \multicolumn{1}{c}{ Stations } \\
\hline 1 & HON and KAK \\
2 & HON and SJG \\
3 & HON and HER \\
4 & KAK and SJG \\
5 & KAK and HER \\
6 & SJG and HER \\
\hline
\end{tabular}

[46] Denote by $\underline{D}_{s, 8, W}, \underline{D}_{s, 9, W}, \underline{D}_{s, 10, W}$ the details with the constant periodic component removed, as described by Jach et al. [2006, paragraph 42]. Thus,

$$
R_{s, W}=\underline{D}_{s, 8, W}+\underline{D}_{s, 9, W}+\underline{D}_{s, 10, W}
$$

is the WISA-based preindex.

[47] We treat the preindex $I_{S}$ defined in (5) and WISA preindex defined in (6) as functional data in UT. Let,

$$
\begin{gathered}
\rho\left(s_{1}, s_{2}\right)=\operatorname{ccorr}\left(I_{s_{1}}, I_{S_{2}}\right), \\
\rho_{W}\left(s_{1}, s_{2}\right)=\operatorname{ccorr}\left(R_{s_{1}, W}, R_{s_{2}, W}\right)
\end{gathered}
$$

be the canonical correlations between preindexes at stations $s_{1}$ and $s_{2}$ computed using improved methodology and the WISA approach.

[48] Since the index of the storm activity is designed to capture global storm signature, the correlation between preindexes at different stations should be higher for the method that removes daily periodic component in a more efficient way. Figures 8-12 contain the canonical correlations for different combinations of stations (see Tables 3-5) during various periods of time. One can see that correlations for our proposed method $\rho\left(s_{1}, s_{2}\right)$ (star) are systematically higher than the correlations for WISA preindex $\rho_{W}\left(s_{1}, s_{2}\right)$ (circle).

[49] In order to check the effect of the averaging over all stations we constructed an alternative preindex, $I_{s}{ }^{*}$, where no averaging over all stations was performed, i.e., skipped step 2. The canonical correlations for this method are labeled with crosses in Figures 8-12. In most cases the resulting correlations are lower than the ones for WISAbased preindex. Therefore, we conclude that averaging over all stations effectively extracts storm signature.

\section{Conclusions}

[50] We propose an improved procedure for removing the daily periodic component which uses statistical filtering

Table 4. Combinations of the Second Set of Four Stations Used to Compare Methodologies

\begin{tabular}{cl}
\hline Combination & \multicolumn{1}{c}{ Stations } \\
\hline 1 & HON and KAK \\
2 & HON and SJG \\
3 & HON and TAN \\
4 & KAK and SJG \\
5 & KAK and TAN \\
6 & SJG and TAN \\
\hline
\end{tabular}

Table 5. Combinations of the Third Set of Six Stations Used to Compare Methodologies

\begin{tabular}{cl}
\hline Combination & \multicolumn{1}{c}{ Stations } \\
\hline 1 & HON and KAK \\
2 & HON and SJG \\
3 & HON and MBO \\
4 & HON and TAN \\
5 & HON and PHU \\
6 & KAK and SJG \\
7 & KAK and MBO \\
8 & KAK and TAN \\
9 & KAK and PHU \\
10 & SJG and MBO \\
11 & SJG and TAN \\
12 & SJG and PHU \\
13 & MBO and TAN \\
14 & MBO and PHU \\
15 & TAN and PHU \\
\hline
\end{tabular}

techniques and functional principal component analysis procedures. As an initial step we use multiresolution analysis to isolate the daily periodic component. To extract the storm signature we use the data from multiple stations. Principal component approach is applied to remove the nonconstant daily variation. Our procedure produces an index which is cleaner than the WISA and the Dst both of which contain significant residual daily variation.

[51] Functional canonical correlations were used to compute a quantitative measure of the level of improvement. We showed that there is a significant improvement from existing WISA and Dst, since WISA index is statistically indistinguishable from Dst. We conclude that our proposed methodology produces an index that isolates the global storm activity in a more efficient and cleaner way.

[52] The method of deconvoluting the daily variation in the presence of a storm offers a potential tool to study its temporal and spacial behavior. Follow-on research will refine the technique of the analysis of the dynamic daily variation to study the relationship between the $\mathrm{Sq}$, storm dynamo, and partial ring currents. Such an analysis is beyond the intended scope of this contribution which emphasizes isolating global rather than local features.

[53] Acknowledgments. Research supported by NSF grants DMS0413653 and DMS-0804165. Data were provided by USGS via the global network of observatories INTERMAGNET.

[54] Zuyin $\mathrm{Pu}$ thanks Wenyao $\mathrm{Xu}$ and another reviewer for their assistance in evaluating this paper.

\section{References}

Anderson, T. W. (1984), An Introduction to Multivariate Statistical Analysis, John Wiley, New York.

Blanc, M., and A. D. Richmond (1980), The ionospheric disturbance dynamo, J. Geophys. Res., 85, 1669-1686.

Chen, G.-X., W.-Y. Xu, A.-M. Du, Y.-Y. Wu, B. Chen, and X.-C. Liu (2007), Statistical characteristics of the day-to-day variability in the geomagnetic Sq field, J. Geophys. Res., 112, A06320, doi:10.1029/ 2006JA012059.

Jach, A., P. Kokoszka, J. Sojka, and L. Zhu (2006), Wavelet-based index of magnetic storm activity, J. Geophys. Res., 111, A09215, doi:10.1029/ 2006JA011635.

Leurgans, S. E., R. A. Moyeed, and B. W. Silverman (1993), Canonical correlation analysis when the data are curves, J. R. Stat. Soc., Ser. B, 55, $725-740$.

Percival, D. B., and A. T. Walden (2000), Wavelet Methods for Time Series Analysis, Cambridge Univ. Press, Cambridge, U. K.

Ramsay, J. O., and B. W. Silverman (2005), Functional Data Analysis, Springer, New York. 
Xu, W.-Y., and Y. Kamide (2004), Decomposition of daily geomagnetic variations by using method of natural orthogonal component, J. Geophys. Res., 109, A05218, doi:10.1029/2003JA010216.

Xu, Z., L. Zhu, J. Sojka, P. Kokoszka, and A. Jach (2008), An assessment study of the wavelet-based index of magnetic storm activity (WISA) and its comparison to the Dst index, J. Atmos. Sol. Terr. Phys., 70, 15791588, doi:10.1016/j.jastp.2008.05.007.
Zhu, L., Z. Xu, J. Sojka, R. Schunk, P. Kokoszka, and A. Jach (2007), Are the Dst stations sufficient for describing storm-time enhancements?, technical report, Utah State Univ., Logan.

P. Kokoszka and I. Maslova, Department of Mathematics and Statistics, Utah State University, Old Main Hill 3900, Logan, UT 84322, USA (Piotr.Kokoszka@usu.edu; Inga.Maslova@aggiemail.usu.edu)

J. Sojka and L. Zhu, Department of Physics and Center for Atmospheric and Space Science, Utah State University, Old Main Hill 4405, Logan, UT 84322, USA. (zhu@cc.usu.edu; fasojka@sojka.cass.usu.edu) 